Revista Educación 25(1): 103-110, 2001

\title{
LA EVALUACIÓN DE LAS PERSONAS CON AUTISMO
}

\author{
Ronald Soto Calderón
}

Resumen: Este artículo pretende brindar elementos básicos que deben ser utilizados en el desarrollo de procesos de evaluación de personas con autismo, la idea es ir dando sentido al diagnóstico realizado por los profesionales, $e$ ir desarrollando estrategias compartidas tanto por docentes (principalmente de Educación Especial) como a psicólogos y otros profesionales involucrados en el trabajo de personas con autismo, de tal manera que se le dé la importancia que se persigue con el trabajo en equipo. Este artículo es una primera parte donde se abordan conceptos y aspectos generales del diagnóstico, posteriormente se hará referencia a técnicas y métodos específicos utilizados en el diagnóstico de personas con autismo.

\section{Introducción}

La medición tanto psicológica como pedagógica de las personas con autismo, es una tarea muy compleja y a la vez sujeta a error.

Sin embargo, medir la conducta de estas personas es indispensable cuando se quiere avanzar tanto en la ciencia psicológica Como en la pedagógica. Por esta razón, se hace necesario el uso de métodos de medida confiables y válidos que permitan una adecuada evaluación y tengan como resultado un apropiado planteamiento terapéutico (tanto en el ámbito psicológico como pedagógico), y se favorezca el buen desarrollo adaptativo y la calidad de vida de la persona con autismo.

Si lo anterior es un punto clave para la mayoría de la población, lo es más para las personas con autismo y otras discapacidades, si se procura, como es la política actual, que todas las personas tengan las mismas oportunidades en los diferentes campos del quehacer humano.

Desde que Kanner en 1943 realizara la descripción inicial del síndrome del autismo, mucho se ha hablado de la presencia de capacidad intelectual entre la población de personas con autismo (Soto, 1996). No obstante, estudios posteriores presentaban resultados contradictorios a la posición anterior, ante lo cual, muchos investigadores acotaban que este comportamiento era debido a inhibiciones de conducta o de aspectos emocionales. Al respecto Revière (1983) comenta que este hace referencia a lo que él denominara "el núcleo de la competencia intacta y la actuación inhibida". 


\section{La evaluación del síndrome del autismo}

Cuando se abordan los temas de evaluación y diagnóstico en autismo, se encuentran con frecuencia afirmaciones por parte de diferentes autores en cuanto a ser un síndrome difícil de diagnosticar, una cuestión problemática o una labor inasequible debido a lo complicado de sus características e identificación de las mismas.

Partiendo de la anterior descripción, es importante aclarar que la evaluación del autismo será abordada desde dos enfoques diferentes, pero complementarios: la evaluación realizada del maestro y la evaluación llevada a cabo por el equipo responsable de atender a la persona con autismo. Es necesario aclarar que este es un punto de vista personal, lo cual puede no dejar satisfechas a otras personas. Sin embargo, se considera que estos dos enfoques interactúan y por lo tanto el enriquecimiento dado es muy necesario y a la vez muy productivo.

Es importante, según anota Alpern (1967), tomar en cuenta la posibilidad de la medición psicológica del autismo. Pues antes de este año, algunos profesionales entre ellos Ferster y DeMyer (1961) y Ferster y otros (1962) se habían dedicado a realizar estudios sobre el tipo de reforzadores a los que tenían preferencia las personas con autismo, también en cuanto a la rapidez de aprendizaje.

Otros autores, entre ellos Davison (1964), Lovaas y otros (1965), Lovaas (1966); realizaron otras evaluaciones conductuales aplicadas a niños con autismo, incluidos en programas de tratamiento conductual.

Todas estas evaluaciones suministraron medidas sobre la existencia o ausencia de comportamientos específicos; entre ellos el habla psicótica, el juego, la conducta social, la conducta estereotipada, y otras conductas presentes en las personas con autismo.

Por otro lado, existe el problema de que los diagnósticos del Síndrome Autista en general han sido poco rigurosos y, a la vez, inconsistentes. A menudo se han utilizado listas de diagnóstico en investigación y en la clínica, pocos ítemes tienen en común (aproximadamente $35 \%$ ), los cuales la mayoría de las veces dan correlaciones moderadas cuando se aplican a los mismos niños (DeMyer et al., 1971).

También se debe anotar que las valoraciones clínicas del autismo muestran un grado de acuerdo muy bajo, según anota Rimland (1971), cuando un profesional da un diagnóstico de autismo, la probabilidad de que otro profesional experimentado también lo dé, es de aproximadamente 0,25\%.

Es importante aclarar, la problemática de diagnosticar a la persona con autismo. En primer lugar se presenta el problema de la definición del autismo, la cual continúa siendo poco precisa: los expertos tienen dificultades para ponerse de acuerdo con las características conductuales necesarias para diagnosticar el autismo, cuáles son los síntomas generales y cuáles son los específicos. Rivière (1985) anota que la razón de esta problemática estriba en la estructura difusa del síndrome, pues hay elementos más típicos que otros, y en general, no todos presentan el mismo grado de representatividad.

Un segundo aspecto por tomar en cuenta en la dificultad del diagnóstico es la heterogeneidad de la población autista, pues, en algunos casos, el autismo aparece solapado con otros trastornos orgánicos y, otras veces, el autismo comparte rasgos esenciales con alteraciones de diferente índole y además, se da el caso de que el autismo puede ir o no asociado con un retraso mental (Wing y otros, 1989; Frith, 1991).

Otro aspecto importante por tomar en cuenta, es la inexistencia de instrumentos adecuados para medir y definir de un modo preciso y concreto las alteraciones y las deficiencias características del síndrome autista. Al respecto Freeman et al., (1981:25) se quejan de que a pesar de la mucha investigación desarrollada en los últimos años desde la descripción del síndrome del autismo 
“... no se haya establecido aún el método objetivo para cuantificar conductas específicas patognomónicas.”

Lo anterior ha provocado que muchos investigadores no se preocupen por un diagnóstico claro y concienzudo de las personas con autismo, y basan su diagnóstico, la mayoría de las veces, en la subjetividad o en el llamado "ojo clínico". Situación considerada poco profesional, pues se considera necesario para poder elaborar y aplicar un buen plan de intervención, contar con un diagnóstico profesional y un análisis detallado de cada persona y de la calidad de su funcionamiento, y destacar de esta manera, los aspectos relevantes para un buen diseño en el aspecto educativo.

Esto ocurre, porque es muy difícil definir objetivamente las conductas, y además, porque la mayoría de las veces se da mucha importancia a los informes de las padres y no se utilizan las observaciones directas de las personas que manifiestan conductas que hacen suponer el padecimiento del síndrome autista. También se parte de descripciones previas del autismo, las cuales de alguna forma han cuantificado posibles conductas que caracterizan a las personas con autismo, pero en las que no ha sido posible hacer una descripción del síndrome.

De esta manera, se puede decir que las personas con autismo pueden ser evaluadas, siempre y cuando se haga un uso adecuado de los métodos, técnicas y procedimientos favorecedores de una aproximación diagnóstica clara y con criterio, tomando como pautas los signos identificados en técnicas y métodos confiables.

Para poder cumplir con este requisito, al ser el Síndrome un conglomerado de síntomas, sería preciso diseñar y emplear una escala que incluya muchas de las conductas, y en cuya formulación se hayan tomado en cuenta a muchas personas con este síndrome. No obstante, hay que señalar que las conductas mostradas por las personas con autismo varían ampliamente, y por lo tanto, el problema se complica.

\section{Principios generales del modelo de evaluación}

El modelo a desarrollar aquí presenta entre sus principios los siguientes aspectos:

1. A las personas con autismo se les pueden aplicar pruebas. Esto hace referencia a que, desde la descripción de Kanner (1943) se consideró que la capacidad intelectual de las personas con autismo era normal, y la mayor parte de los periodos de atención cortos, problemas de contacto ocular, y otros, los cuales de alguna forma son incompatibles con los requisitos conductuales mínimos necesarios para poder realizar, en forma satisfactoria, las pruebas psicológicas estandarizadas. Esta posición de Kanner de los niños con autismo inteligentes, pero no testables, fue aceptada sin reparos, hasta muy entrados los años sesenta. Al respecto Rivière (1983) hace referencia al mito de la competencia intacta y la actuación inhibida. Como ya se había mencionado anteriormente, los estudios de Alpern (1967) demostraron ampliamente la posibilidad de la medición psicológica de las personas con autismo. Para Alpern, los fracasos de los intentos anteriores en la determinación de las capacidades cognoscitivas de las personas con autismo podía explicarse: pues se consideraba que las pruebas utilizadas eran demasiado difíciles. Por esto se propone el uso de pruebas diseñadas para bebés, para determinar las capacidades de las personas con autismo, comprobando, por lo tanto, que estas personas son testables siempre y cuando se utilicen los instrumentos de evaluación adecuados.

2. Otro aspecto a tomar en cuenta es el del principio de normalización. Al respecto comenta Wolfensberger (1980) es el uso de medios y medidas lo mas 
culturalmente normativos para poder establecer y posibilitar conductas que sean lo mas culturalmente normativas posible.

3. La evaluación del autismo debe investigar y analizar las características del comportamiento individual en su interacción con el ambiente en que se desenvuelve, o sea es de carácter ideográfico y tiene carácter interactivo.

4. El fin último de la evaluación será, por lo tanto, describir en forma empírica las características del niño con autismo con el fin de que posteriormente se pueda llevar a cabo, la intervención. De esta manera, la evaluación va a hacer posible la planificación pedagógica en forma individual y, por lo tanto, cualquier método utilizado debe tener una traducción inmediata en la práctica. De esta manera debe servir para la ubicación adecuada de la persona con autismo dentro de los curricula concretos planteados para aula. Se concluye con Tamarit (1985) que la idea de la evaluación y el tratamiento son dos aspectos de un mismo proceso. De esta manera, entre la evaluación y el tratamiento se establece una dinámica continua de contrastación de hipótesis.

5. Un aspecto digno de mencionar es el de la verificación posterior o, seguimiento, el cual debe darse en forma sistemática de tal manera que permita determinar la evolución de la persona con autismo y poder contrastar y revisar los resultados obtenidos.

6. Por otro lado, la evaluación del autismo debe adoptar un enfoque prescriptivo, el cual no solamente sugiere la capacidad de hacer cosas de una persona con autismo, sino que muestra lo que la mayoría de estas personas pueden hacer posteriormente.
7. Por último, la evaluación del autismo debe ser de carácter multiaxial. De esta manera, paralelo a la evaluación psicopedagógica, se debe tener en cuenta otros indicadores codificados en ejes independientes, los cuales determinan la naturaleza y el pronóstico del síndrome. Entre ellos se pueden mencionar entre otros: si la persona presenta algún asociado de retraso mental, las condiciones médicas y los trastornos neurológicos o la situación psicosocial.

\section{Modelo integrador}

La evaluación de la persona con autismo debe partir del principio de un sistema de relaciones que interactúan y pueden ser medidos independientemente, aunque la forma de actuación sólo es comprensible al verla como unidad, producto de las interacciones de los elementos.

Por lo tanto, la evaluación a realizar deber ser lo mas completa y exhaustiva posible del comportamiento de la persona con autismo, mediante una gama de conductas, dándose sin embargo, la especificidad situacional y de contenido, siempre con la posibilidad de que la información recogida tenga un carácter multimodal y las fuentes de información sean variadas.

En este modelo se considera la evaluación como una parte del proceso terapéutico, constituyendo el primer paso para la elaboración de todo programa educativo. La evaluación tiene en cuenta no solo las características de la persona con autismo, sino también las del medio en que se desarrolla.

\section{Evaluación de la persona con autismo}

Se puede dar un análisis cuantitativo, y se aplican una serie de técnicas e instrumentos de manera objetiva y cuantificante. 
Este apartado incluye tres tipos de análisis, a saber:

1. El análisis normativo. En este caso, se usan estos procedimientos para evaluar las características representativas del desarrollo madurativo y determinar el nivel evolutivo de la persona con autismo. También lo usa el maestro al comparar el rendimiento de la persona con autismo con el resto del grupo.

2. El análisis con base en criterios y curriculum. Permite conocer la situación del niño respecto a un criterio de dominio que se ha establecido para una tarea, un programa educativo, en general se refiere al currículum.

3. El análisis comparativo intrasujeto. La comparación se hace en función de puntuaciones obtenidas por el mismo sujeto.

La evaluación cuantitativa se hace necesaria pues permite:

A. Evaluar productos y rendimientos.

B. Determinar el nivel actual de la persona con autismo y los progresos al realizar las tareas en forma autónoma.

Sin embargo, la evaluación cuantitativa por sí sola no es suficiente para dar una visión completa del niño y, por esto, se debe complementar con la evaluación cualitativa.

El análisis cualitativo no utiliza ningún método concreto de medida y, quizás este sea uno de los problemas que presenta. Para poder hacerlo es necesario el uso de la observación y el conocimiento del desarrollo del niño normal y de la persona con autismo, así como un conocimiento epistemológico.

La evaluación cualitativa permite conocer el nivel de desarrollo potencial, determinar el nivel de ayuda e identificar los procesos y estrategias utilizados por la persona al realizar una tarea.
La evaluación no solo debe contener aquello que la persona con autismo es capaz de hacer por si sola, si no también aquellas tareas que realiza con la ayuda dada. Al respecto anota Vigotsky (citado por Rivière, 1983, p. 53) “... la distancia entre el nivel actual de desarrollo determinado por la capacidad de resolver independientemente un problema, y el nivel de desarrollo potencial, determinado a través de la resolución de un problema bajo la guía de un adulto o en colaboración con otro más capaz."

La evaluación ha de tener en cuenta, no solamente las características del niño, sino también las del entorno en que se desarrolla, como son: la familia, la escuela e incluso el aspecto social. Para obtener información al respecto, se recurrirá al análisis cualitativo principalmente y como técnica importante la observación, lo cual se podrá llevar a cabo, por medio de registros sistemáticos, diarios, fotografías, vídeos y otros.

\section{Evaluación en el aula}

No se considera al educador o terapeuta como el único responsable de la evaluación dentro del aula, ya que se hace necesaria la participación de otros profesionales como el orientador, el psicólogo y otros. Lo ideal sería una evaluación en conjunto para la elaboración de un cuadro completo de los trastornos funcionales, de los problemas conductuales de la persona con autismo, y de sus capacidades.

Sin embargo, este trabajo conjunto no es siempre posible y, por lo tanto, se debe recurrir a las anotaciones y observaciones que han realizado los maestros, las cuales pueden constituir una base para un programa de enseñanza. La situación del maestro en este caso es ventajosa por el tiempo que pasa con la persona con autismo. Además de la formación profesional y la experiencia que le han dado otros niños. 
La evaluación del maestro es continua y constante, procura conocer y comprender a las personas con autismo, pero no da interpretaciones teóricas, esto permite una retroalimentación sobre el rendimiento del niño y el logro de los objetivos planteados. De esta manera, la descripción hecha por el educador es más global y contextualizada, por lo tanto mas pormenorizada.

No se puede olvidar que toda evaluación es un proceso para la toma de decisiones, por lo tanto, se debe tener muy claro a qué proceso de decisión sirve la evaluación, pues este determina desde el principio la naturaleza de la evaluación.

En general se puede decir que la evaluación del educador o terapeuta esta compuesta por las siguientes fases:

1. Recoger y analizar la información (observación), cuestionarios, entrevistas.

2. Describir e identificar.

3. Hipotetizar. ¿Plantear preguntas?

4. Seleccionar y aplicar técnicas (análisis funcional, observación sistemática).

5. Elaborar resultados.

6. Tomar decisiones (intervenir o prevenir).

\section{Evaluación por parte del equipo}

Esta evaluación exige un seguimiento sistemático de la evolución de la persona con autismo, pero no es un proceso continuo sino mas bien puntual. Tiene un carácter más interpretativo, busca las causas y determina la forma de actuar de estas personas. La información que dan sirve al maestro para decidir en qué fijarse, como interpretar la información y como utilizarla para la elaboración del programa de intervención.

La evaluación de la persona con autismo requiere el uso de diferentes fuentes de información entre ellas: la observación tradicional, la entrevista, instrumentos estandarizados y específicos, técnicas de observación directa, escalas de conducta (diagnóstica y descriptiva).

El obtener la información por medio de técnicas tiene una doble finalidad, en primer lugar, examinar los distintos tipos de funciones mentales mediante el empleo de tests basados en criterios normalizados del desarrollo, y en segundo lugar, observar y describir.

La evaluación del equipo sigue los siguientes pasos:

1. Recolección de datos.

2. Observación y aplicación de pruebas.

a. Nivel intelectual

b. Desviación-Desarrollo social

c. Desviación-Comunicación y lenguaje

d. Invarianza ambiental

3. Toma de decisiones
a. Clasificar
b. Predecir
c. Orientar

\section{Evaluación como punto de partida de la intervención}

Una vez que la persona con autismo ha sido evaluada, se hace necesaria la intervención, con el fin de favorecer un desarrollo positivo de su conducta. Por lo tanto, se requiere la planificación y aplicación de una determinada intervención, que en el aspecto educacional adquiere la forma de "plan correctivo" o "programa educativo". En este se incluyen los objetivos generales por alcanzar de la persona con autismo, los métodos y las técnicas por emplear para poder enseñar y cómo evaluar. El programa debe ser diseñado en forma individual y es responsabilidad del maestro. 


\section{Escalas e instrumentos de evaluación}

Hay algunos instrumentos que pueden ayudar en la labor por realizar con personas con autismo. A continuación se mencionan algunas de fácil manejo:

1. "Escala de evaluación de la Conducta (EEC)", Kozloff (1980).

2. “¿Cómo enseñar a mi hijo?”, Baker (1980).

3. "Guía Portage de educación preescolar", Bluma et al. (1978).

\section{Conclusiones}

Como se ha desarrollado en el artículo, es posible y necesaria la evaluación de las personas con autismo. Por otro lado la evaluación realizada por el educador tiene como finalidad comprender y conocer a la persona con este síndrome, mientras que la evaluación del equipo tiene un carácter más interpretativo, busca las causas que determinan la forma de actuar de la misma.

Toda evaluación debe ser global, cuidadosa y detallada, ya que como se anotó, una de las principales características de las personas con autismo es la carencia de uniformidad en su rendimiento. Además, la evaluación del autismo exige hacer uso constante de dos niveles de análisis, el cuantitativo y el cualitativo.

Muchos son los aspectos que han sido abordados, sin embargo, todavía queda mucho qué investigar en cuanto a la evaluación de la persona con autismo.

\section{Referencias bibliográficas}

Alpern, G.D. "Measurement of undestable autistic children ". Journal of Abnormal Psychology, 72, 478. 1967.
Baker, B.L. y otros. ¿Cómo enseñar a mi hijo? Madrid: Pablo del Rio. 1980.

Bluma, S. y otros. Portage Guide. Winsconsin: CESA (Adaptacion TEA). 1978.

Davison, G.C. "A social learning therapy programme with an autistic child." Behavior Research and Therapy. 2: 149-160. 1964.

DeMyer, M. K. et al. "Social adaptative behaviors of autistic children measured in a structural psychiatric interview. Churchill(ed): Autism: Proceedings of Intervention. The Indiana University. Indiana: Springfield, III. 1971.

Ferster, C. B. y DeMyer, M.K. "The development of performances in autistic children in an automatically controlled enviroment." Journal of chronic Diseases, 13:312-354. 1961.

Ferster, C.B. y otros. A method for the experimental analisis of the behavior of autistic children." American Journal of Orthopsychiaty. 32:89-98. 1962.

Freeman, B.J. et al. "The Behavior Observation Scale for Autism. Initial methodology and preliminary findings on 89 children." J. of the American Academy of Child Psychiatry, 17: 576-588. 1981.

Freeman, B.J. et al. "Behavior assessement of the Syndrome of Autism: Behavior Observation System". Journal of the American Academy of Child Psychiatry. 23:588-594. 1984.

Frith, Uta. Autismo. Explicación al enigma. Madrid: Editorial Alianza. 1991.

Kozloff, M.A. Escala de Evaluación de la Conducta (E.E.C). En: El aprendizaje y la 
conducta en la infancia. Barcelona: Fontanella. 1980.

Lovaas, O.I. y otros. "Experimental studies in childhood schizophrenia." Behavior Research and Therapy, 2:67-84. 1965.

Lovaas, O.I. "Adquisition of imitative speech by schizophrenia children." Science. 151: 705-707. 1966.
Rivière, A. "Interacción y símbolo en autistas". Infancia y Aprendizaje. (22), 3. 1983.

Tamirit, J. (1985). "Evaluación y tratamiento: dos aspectos de un mismo proceso". Madrid: III Congreso Nacional de AETAPI.

Wing, L. et al. (1989). Autismo Infantil: Aspectos médicos y educativos. Madrid: Editorial Santillana. 